\title{
Attachement père-cadet et qualité des relations fraternelles cadet-aîné
}

\section{Father-child attachment and quality of the sibling relationships}

\author{
S. Pinel-Jacquemin ${ }^{*}, 1$, C. Zaouche-Gaudron ${ }^{2}$, \\ O. Troupel-Cremel ${ }^{3}$
}

UFR de psychologie, équipe «Milieux, groupes et psychologie du Jeune-Enfant», laboratoire psychologie du développement et processus de socialisation, université Toulouse II-Le-Mirail, 5, allées Antonio-Machado, 31058 Toulouse cedex 9, France

Reçu le 22 janvier 2009 ; accepté le 3 août 2009

\section{Résumé}

L'objectif de cette étude vise à établir un lien entre la relation d'activation (ou d'attachement) père-enfant et la qualité des relations fraternelles chez des enfants d'âge scolaire, en tenant compte de l'évolution théorique et méthodologique, de cet attachement spécifique. Cinquante-sept pères ont répondu au questionnaire Q1 d'ouverture au Monde (QOM) (Paquette et Zaouche-Gaudron, 2003) qui définit l'attachement ou la relation d'activation, père-enfant selon deux axes: la stimulation (sociale, cognitive et affective) et le contrôle de l'enfant. Le questionnaire des relations fraternelles des jeunes enfants [Troupel, O., 2006. Attachement fraternel, styles de relations et des interactions de tutelle au sein des fratries de jeunes enfants : effet modulateur de la représentation des relations fraternelles de l'aîné. Thèse de doctorat nouveau régime, université de Toulouse-Le-Mirail] renseigne la qualité des relations fraternelles. Les cadets bénéficiant d'une relation sécurisée, c'est-à-dire à la fois stimulante et contrôlante, avec leur père, s'entendent mieux avec leur aîné que les autres enfants, en particulier que ceux qui ont beaucoup de contrôle et peu de stimulation de la part de leur père. L'hypothèse selon laquelle la stimulation envers les pairs du cadet par le père influencerait directement

\footnotetext{
* Auteur correspondant.

Adresse e-mail : steph.jacquemin@worldonline.fr (S. Pinel-Jacquemin).

${ }^{1}$ Doctorante. Thèmes de recherche: l'attachement parents-enfants, le système familial, les relations intrafamiliales, conjugales, coparentales, parents-enfants et fraternelles.

${ }^{2}$ Professeur en psychologie du développement, université Toulouse II-Le-Mirail. Thèmes de recherche : le développement socio-affectif du jeune enfant, la paternité en situation de précarité, l'identité sexuée du jeune enfant.

${ }^{3}$ Docteur en psychologie du développement et ATER, université Toulouse II-Le-Mirail. Thèmes de recherché : relations fraternelles, attachement fraternel, apprentissage cognitif du jeune enfant.
} 
la qualité de la relation fraternelle n'est cependant pas confirmée. D'autres résultats sur l'attachement et les relations fraternelles sont discutés.

(C) 2009 Publié par Elsevier Masson SAS pour la Société française de psychologie.

Mots clés : Attachement père-enfant ; Relations fraternelles ; Approche systémique ; Stimulation paternelle ; Contrôle paternel

\begin{abstract}
The aim of this study is to establish a link between the father-child attachment and the quality of the sibling relationships for school-age children, by taking into account the theoretical and methodological evolution of this specific attachment. Fifty-seven fathers answered the Questionnaire of Opening up to the World (Paquette et Zaouche-Gaudron, 2003), which defines the father-child attachment (or activation) on two axes: the stimulation (social, cognitive and emotional) and the paternal control exercised over the child. The Questionnaire "Sibling Relationships in Young Children" [Troupel, O., 2006. Attachement fraternel, styles de relations et des interactions de tutelle au sein des fratries de jeunes enfants: effet modulateur de la représentation des relations fraternelles de l'aîné. Thèse de doctorat nouveau régime, université de Toulouse-Le-Mirail] gives insight into the quality of sibships. Children, with a secure attachment to their father, demonstrate a higher level of cooperation with their elder sibling than the other children, in particular those which receive excessive control and little stimulation from their fathers. The hypothesis that the stimulation of the second-born child by the father on the peer side is directly linked to the quality of the sibships is not confirmed. Other results on father-child attachment and sibships are discussed. (C) 2009 Published by Elsevier Masson SAS on behalf of Société française de psychologie.
\end{abstract}

Keywords: Child-father attachment; Sibling relationships; Systemic perspective; Paternal stimulation; Paternal control

\title{
1. Introduction
}

L'attachement a été initialement défini par Bowlby (1969) comme étant, pour l'enfant, un besoin social primaire et inné d'entrer en relation avec sa mère. L'enfant utilise celle-ci comme base de sécurité pour ensuite pouvoir explorer son environnement. Par la suite, Bowlby (1973) a reconnu le rôle possible d'autres figures d'attachement et notamment celle du père. Le concept de monotropie (la mère, ou son substitut, comme unique figure d'attachement) a alors fait place à un ensemble hiérarchisé de figures d'attachement, dont la mère resterait l'élément principal. Les travaux de Lamb $(1977,1996)$ confirment ces différences quantitatives entre pères et mères, selon les moments et les contextes d'interaction avec l'enfant : ainsi, lorsque les parents sont tous deux présents, l'enfant recherche davantage la proximité de sa mère. Sur le plan qualitatif, l'hypothèse selon laquelle l'enfant développe le même attachement auprès de son père et auprès de sa mère a été progressivement remise en question. En effet, l'attachement mère-enfant se formerait dans un contexte de soins (Bowlby, 1969), alors que l'attachement père-enfant se construirait plutôt au travers des jeux. Les travaux sur la contribution spécifique du père dans le développement de l'enfant le désignent comme étant un «catalyseur de prise de risques » (Kromelow et al., 1990), un «agent de socialisation» (Le Camus, 2000), un «agent de subjectivation et de dynamisation» (Le Camus in Le Camus et al., 1997) ou encore un «pont social» entre l'enfant et les autres (Zaouche-Gaudron, 2002). Selon Paquette (2004), le lien d'attachement père-enfant devrait être qualifié de «relation d'activation». Ainsi, le père formerait pour l'enfant une base d'activation complémentaire de la base de sécurité constituée par la mère. L'enfant chercherait alors «protection» auprès de sa mère et «stimulation» auprès de son père (Grossmann et al., 
2002 ; Le Camus, 2000 ; Miljkovitch de Heredia et al., 1998 ; Paquette, 2004). Grossmann et al. (2002) rejoignent cette conception et parlent d' «exploration sécurisée » qui diffère, selon eux, du concept de base de sécurité, par le rôle différent et complémentaire joué par les deux parents durant l'activité exploratoire de l'enfant.

Parallèlement à ce changement de perspective, se développe actuellement un courant théorique qui prône l'intégration de la théorie de l'attachement dans la théorie du système familial (Forman et Davies, 2005 ; Hill et al., 2003 ; Kozlowska et Lesley, 2002). Déjà, en 1990, Marvin et Stewart soulevaient l'intérêt de considérer l'influence exercée sur l'attachement par les autres soussystèmes familiaux qui caractérisent l'environnement de vie principal de l'enfant. Il est composé d'au moins deux enfants et est influencé par de multiples facteurs tels que les « caractéristiques morphologiques » (taille, écart d'âge, composition sexuée) de la fratrie, mais aussi par le contexte familial (conflit conjugal, traitement parental différentiel, etc.), les caractéristiques individuelles des enfants (comme leur tempérament), ou encore les représentations que les parents ont de la fratrie, du rang qu'ils ont eux-mêmes occupé dans leur propre fratrie et par les identifications qui en découlent (Bourguignon, 1999).

Les différentes études portant sur le rôle du rang de naissance, de la composition sexuée (Abramovitch et al., 1980; Stewart, 1983; Dunn et Kendrick, 1979) et de la taille de la fratrie (Camdessus, 1998) sur la qualité des relations fraternelles ont amené des résultats très contradictoires. En revanche, un consensus s'est fait autour de la question de l'écart d'âge et il a clairement été démontré que la qualité des relations fraternelles était meilleure pour des frères et sœurs ayant moins de deux ans ou plus de quatre ans d'écart (Almodovar, 1981; Angel, 1996). La rivalité fraternelle serait donc à son apogée entre deux et quatre ans d'écart d'âge entre deux frères et/ou sœurs. Dans tous les cas, c'est toujours ce que l'aîné dit de ses relations avec son cadet qui est considéré et non ce que dit le cadet de sa relation avec son aîné.

L'univers des relations fraternelles a d'abord été exploré, sous l'angle vertical (conflit, rivalité par rapport à l'objet maternel, etc.) par la psychanalyse (Lacan, 1938 ; Kaës, 1993), puis, dans sa dimension horizontale (la fratrie comme système horizontal autonome) par les psychologues du développement (Almodovar, 1981). Welz (1984), Widmer (1999) et Troupel (2006), quant à eux, proposent une typologie des relations fraternelles relatifs à deux axes : l' «opposition » et la « coopération» : en effet, il existe des relations fraternelles consensuelles (avec à la fois beaucoup de coopération et peu d'opposition), ambivalentes (avec à la fois beaucoup de coopération et beaucoup d'opposition), conflictuelles (peu de coopération et beaucoup d'opposition) et de faible implication (peu de coopération et peu d'opposition).

L'imbrication des deux dimensions, verticale et horizontale, apparaît cependant nécessaire pour mieux en comprendre l' articulation (Almodovar, 1981). Ainsi, la relation parents-enfants et la relation fraternelle représenteraient-elles non seulement deux microsystèmes, mais aussi un mésosystème avec son jeu de relations interdépendantes. Quatre études ont été recensées qui ont analysé cette imbrication. Tout d' abord, celles de Bosso (1986), de Teti et Ablard (1989) et de Coutu, Provost et Pelletier (1995), qui, se concentrant sur le lien entre l'attachement mère-enfant et la qualité des relations fraternelles, ont montré qu'effectivement la classification d'attachement, sécurisé versus insécurisé, était utile pour comprendre les différences individuelles dans l'implication affective des jeunes fratries. Puis, celle de Volling et Belsky (1992) qui est la seule, à notre connaissance, à s'être intéressée à la relation entre l'attachement père-enfant (mesuré avec la procédure de la situation étrange) et la qualité des relations fraternelles, sans toutefois y trouver de liens significatifs. En revanche, ces auteurs ont montré davantage d'interactions prosociales entre les enfants lorsque le père est stimulant avec l'aîné de trois ans pendant les jeux libres et lorsque le père témoigne plus d'affection à son aîné de six ans qu'au cadet. 
Tableau 1

Caractéristiques de la population cadets - aînés - dyades aîné/cadet : nombre, âge et écart d’âge selon le sexe des enfants.

\begin{tabular}{|c|c|c|c|c|c|c|c|c|c|c|c|}
\hline & \multicolumn{3}{|c|}{ Cadet } & \multicolumn{3}{|l|}{ Aîné } & \multicolumn{3}{|c|}{ Dyades } & \\
\hline & $\mathrm{F}$ & G & Tot & $\mathrm{F}$ & $G$ & Tot & $\mathrm{F}-\mathrm{F}$ & $\mathrm{F}-\mathrm{G}$ & G-F & $\mathrm{G}-\mathrm{G}$ & Tot \\
\hline Nombre & 30 & 27 & 57 & 33 & 24 & 57 & 18 & 15 & 12 & 12 & 57 \\
\hline Âge moyen & 5,2 & 5,1 & 5,16 & 7,52 & 7,04 & 7,32 & & & & & \\
\hline Écart d'âge & & & & & & & 2,07 & 2,65 & 1,72 & 2,13 & 2,16 \\
\hline
\end{tabular}

F : fille ; G : garçon ; Tot : total.

Ces résultats rejoignent directement notre problématique puisque nous considérons que c'est dans un contexte stimulant et contrôlant (le contrôle paternel consistant à limiter l'exploration de l'enfant dans le but de le protéger ; «l'exploration sécurisée » de Grossmann et al., 2002) que se crée un lien d'attachement singulier et spécifique entre l'enfant et son père. Cet attachement est source pour l'enfant d'acquisitions de compétences sociales, définies comme une plus grande facilité à gérer les conflits, à respecter les normes et les règles, etc. Ces habiletés sociales servent aux autres relations sociales de l'enfant, et en particulier, celles qu'il rencontre dans son premier milieu de vie, notamment avec ses frères et sœurs. Ainsi, l'attachement père-enfant donnerait les moyens à l'enfant de répondre de manière plus adaptée aux conflits au sein de sa fratrie et lui permettrait de développer des relations fraternelles de meilleure qualité.

Dans cette perspective systémique, l'hypothèse que nous formulons est que l'attachement envers le père influence la qualité des relations des enfants au sein de leur fratrie.

\section{Méthode}

\subsection{Population}

Notre population a été recrutée par bouche-à-oreille sur les critères d'inclusion suivants : famille française, deux enfants entre trois et 12 ans et vivant sous le même toit que leur père, l'écart d'âge entre les enfants ne doit pas dépasser six ans. Elle est constituée de :

- cinquante-sept pères ( $\mu=37,06$ ans, $\sigma=4,28)$ appartenant en majorité $(87,4 \%)$ à la profession catégorie socioprofessionnelle ${ }^{4}$ de niveau 3 (cadres et professions intellectuelles supérieures);

- cinquante-sept aînés ( $\mu=7,32$ ans, $\sigma=1,23)$ et leurs cadets $(\mu=5,16$ ans, $\sigma=0,70)$. Les premiers ont entre cinq ans et cinq mois et 11 ans et trois mois, tandis que les derniers sont tous nés en 1999 ou 2000 (un enfant né en 2001), ce qui fait un âge minimum de trois ans et neuf mois et un âge maximum de six ans.

L'écart d'âge des enfants selon la composition sexuée des dyades se répartit de manière homogène (Tableau 1).

\subsection{Outils}

\subsubsection{Fiche de renseignements}

Une fiche signalétique a été jointe aux questionnaires et envoyée, par courrier ou par mail, aux pères sélectionnés selon le critère d'âge des enfants. Elle permet de repérer les variables

${ }^{4}$ PCS : profession catégorie socioprofessionnelle : remplace les CSP (catégories socioprofessionnelles) depuis 1982. 
concernant non seulement leur identité, mais aussi leur propre rang dans leur fratrie, le nombre et le sexe de leurs enfants ainsi que leur âge et écart d'âge.

\subsubsection{Le questionnaire d'ouverture au Monde (QOM)}

Les pères ont répondu à ce questionnaire concernant leurs relations avec leur cadet. Le choix du cadet nous a paru justifié par le peu d'études s'intéressant à cet enfant précis et la possibilité de disposer, ainsi, d'informations à son égard. Ce mode de recueil de données a été élaboré par Paquette et al. (sous presse) pour pallier le manque d'outils de mesure de l'attachement pèreenfant. Il tient compte de l'ensemble des apports théoriques sur la contribution spécifique du père dans le développement cognitif, social et affectif de son enfant (Nugent, 1991; Yogman et al., 1995 ; état de la question in Le Camus et al., 1997, Le Camus, 2000 ; Zaouche-Gaudron, 2002). Il se focalise sur le versant «exploration/socialisation» de l'attachement plutôt que sur celui de «base sécurisante» relatif à la théorie de l'attachement dans sa version première.

Si pour mesurer l'attachement de l'enfant à sa mère, on a évalué ses comportements de recherche de proximité, on va évaluer ici le degré de stimulation que le père exerce sur son enfant dans des situations de la vie quotidienne, et également par rapport aux adultes, aux pairs, et à son développement cognitif ainsi que le degré de contrôle exercé, que ce soit également lors de situations quotidiennes ou par rapport aux pairs. Une relation d'attachement serait perçue comme «sécurisée », si le père est à la fois stimulant pour son enfant, mais aussi contrôlant, dans le sens où il lui met des limites, sans entraver son autonomie. C'est sous cette hypothèse que Paquette (2004) a proposé le terme de «relation d'activation» pour distinguer ce lien de celui qui unit l'enfant à sa mère.

Ainsi, le questionnaire est composé de 83 items dont les réponses se situent sur une échelle de Likert en six points (d'un : jamais à six : très souvent) et sont répartis en six sous-échelles.

\subsubsection{Sous-échelles de stimulation ( 55 items). Stimulation: «situation» (27 items); stimula-} tion «adultes» (six items); stimulation «pairs» (dix items); stimulation «cognitif» (12 items).

La dimension «stimulation situation» comporte des items concernant la prise de risque (ex : « J'encourage mon enfant lorsqu'il entreprend une activité risquée, comme par exemple : grimper aux arbres, faire de la planche à roulettes, etc...»), les jeux physiques père-enfant (ex : «je fais des jeux de lutte avec mon enfant») et l'exploration proprement dite (ex : «J'invite mon enfant à explorer autour de lui lorsque nous allons à un nouvel endroit (ex : se promener, toucher des objets).

La dimension «stimulation adultes» est mesurée, par exemple, par l'item suivant: «J'encourage mon enfant à s'exprimer devant des étrangers (ex: commander au restaurant, demander une information)».

Celle de «stimulation pairs» s'illustre, par exemple, par : «lorsque mon enfant hésite à parler à un autre enfant de son âge, je l'invite à prendre l'initiative du premier contact».

Pour «stimulation cognitif », les items concernent : la résolution de problèmes ( «J'encourage mon enfant à terminer par lui-même les jeux de puzzle ou d'encastrement») et le langage (« J'utilise des phrases courtes lorsque je m'adresse à mon enfant»).

\subsubsection{Sous-échelles de contrôle (29items). Contrôle: «situation» (25 items); contrôle «pairs» (4 items).}

Pour la dimension «contrôle situation», les items sont par exemple : «Si mon enfant se blesse au parc, je le réconforte, puis nous rentrons à la maison». 
Tableau 2

Fiabilité du QOM - Calcul de l'alpha de Cronbach par sous-échelle.

\begin{tabular}{|c|c|c|c|c|c|c|}
\hline Sous-échelles & Nombre d'items & Moy & Min & Max & Variance & Alpha \\
\hline \multicolumn{7}{|l|}{ Stimulation } \\
\hline Situation & 27 & 4,1 & 2,7 & 5,1 & 0,34 & 0,8728 \\
\hline Adultes & 6 & 3,92 & 3,1 & 4,68 & 0,335 & 0,6143 \\
\hline Pairs & 10 & 3,49 & 2,6 & 4,54 & 0,384 & 0,6603 \\
\hline Cognitif & 12 & 4,06 & 2,7 & 5,09 & 0,834 & 0,6337 \\
\hline \multicolumn{7}{|l|}{ Contrôle } \\
\hline Situation & 25 & 3,46 & 2 & 5,32 & 0,937 & 0,7699 \\
\hline Pairs & 4 & 2 & 1,6 & 2,88 & 0,353 & 0,3239 \\
\hline
\end{tabular}

Moy : moyenne; Min : minimum; Max : maximum.

Et enfin, pour mesurer la dernière dimension «contrôle pairs », un item peut être par exemple : «J'interviens lorsqu'un conflit éclate entre mon enfant et un autre enfant de son âge même si aucun d'eux ne le sollicite».

Les réponses, pour l'ensemble de ces dimensions, sont constituées par une échelle de Likert en six points : d'un : «jamais » à six : «très souvent ». S'il est impossible d'évaluer le comportement énoncé, la réponse «Ne peux évaluer » est prévue. Ainsi, quatre différents types de relations pèrecadet sont obtenus, selon que les niveaux de stimulation et de contrôle sont bas ou élevés : nous les noterons par la suite : $\mathrm{S}+\mathrm{C}+$ (stimulation élevée, contrôle élevé; cette relation correspond à la relation d'activation telle que définie par Paquette, 2004), S-C - (stimulation basse, contrôle bas); $\mathrm{S}+\mathrm{C}-$ (stimulation élevée, contrôle bas) et $\mathrm{S}-\mathrm{C}+$ (stimulation basse, contrôle élevé).

Cet outil a été validé sur 266 pères (207 québécois et 59 français) d'un enfant âgé entre 26 et 69 mois (Paquette et al., sous presse). Pour l'échantillon français, l'alpha de Cronbach (Cronbach, 1951) montre une consistance interne globale acceptable (alpha $=0,64)$. Pour le détail des alphas selon les différentes sous-échelles (Tableau 2).

La stabilité temporelle est établie: les mêmes sujets, interrogés à deux reprises (à six mois d'intervalle), obtiennent $80 \%$ de scores identiques.

Un attachement «activé» père-enfant correspond à une relation à la fois stimulante $(\mathrm{S})$ et contrôlante $(\mathrm{C})$ du père $(\mathrm{S}+\mathrm{C}+)$. C'est la médiane des scores recueillis pour chacun des deux axes «stimulation» et «contrôle» qui nous permet de différencier les pères «stimulants » $(\mathrm{S}+)$ des pères «non stimulants» $(\mathrm{S}-)$, et les pères «contrôlants » $(\mathrm{C}+)$ des pères «non contrôlants » $(\mathrm{C}-)$, cela afin d'éviter d'obtenir une moyenne ayant peu de sens et risquant d'être perturbée par des données aberrantes ou extrêmes.

\subsubsection{Le questionnaire sur les relations fraternelles des jeunes enfants}

Le «questionnaire sur les relations fraternelles des jeunes enfants » (QRF) (Troupel, 2006), permet de mesurer la qualité des relations fraternelles selon les deux axes retenus par Widmer (1999) : la coopération (C) et «l'opposition» (O). Il est renseigné par les cadets (les mères leur expliquent la consigne et lisent les questions aux plus jeunes qui peuvent cocher eux-mêmes leurs réponses). Le QRF est composé de 67 items.

2.2.3.1. Dix-sept items de «coprésence ». Ces items, insérés en début de questionnaire, visent à s'assurer que les deux frères et sœurs passent effectivement du temps ensemble. Ils sont nécessaires afin d'apprécier la qualité de la relation fraternelle.

Exemple : «Es-tu avec ton frère ou ta sœur pour le petit-déjeuner?». 


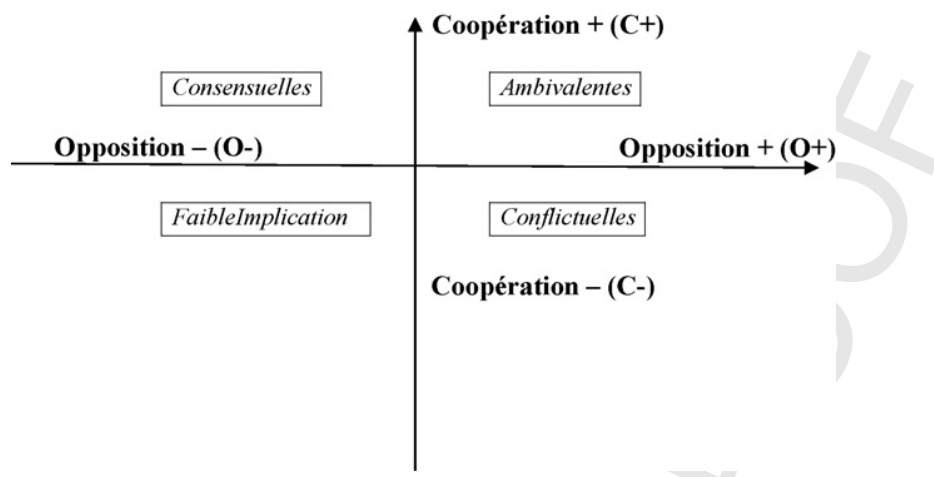

Fig. 1. Typologie des relations fraternelles (Widmer, 1999; Troupel, 2006).

2.2.3.2. Vingt-six items de «coopération» sont répartis en cinq sous-échelles. «Sentiment» (deux items), «partage» (cinq items), «tutelle» (sept items), «complicité » (six items) et «rôle parental» (six items).

Exemple : «Est-ce que tu fais souvent des choses avec ton $\mathrm{F} / \mathrm{S}^{5}$ ? »

2.2.3.3. Vingt-quatre items d'«Opposition» sont, quant à eux, répartis en trois sous-échelles. «Rivalité » (six items), «Différenciation» (neuf items), «Conflit» (neuf items).

Exemple : «Est-ce que ton F/S ${ }^{1}$ t'embête?»

Quatre types de relations fraternelles sont définis selon le schéma dans la Fig. 1.

\subsubsection{Consistance interne et stabilité temporelle $d u Q R F$}

Hormis les deux sous-échelles «complicité » et « rivalité » pour lesquelles la cohérence interne est un peu faible, les échelles et les sous-échelles de ce questionnaire présentent une consistance satisfaisante (les alphas de Cronbach étant compris entre 0,54 et 0,77). L'alpha de Cronbach global est ici de 0,69 .

La stabilité temporelle du QRF est bonne puisque les mêmes sujets, évalués à deux reprises (à six mois d'intervalle), obtiennent $83 \%$ de réponses identiques.

\section{Résultats}

Après avoir présenté les principaux résultats concernant, d'une part, les relations d'activation père-cadet et, d'autre part, les relations fraternelles cadet-aîné, nous verrons quels liens ont pu être établis entre ces deux variables. Dans un premier temps, nous présenterons les statistiques descriptives les plus intéressantes et, dans un second temps, nous exposerons les résultats des différents tests statistiques de comparaison de moyennes.

\subsection{Relations d'«activation » père-cadet}

C'est en raison du choix de la médiane pour distinguer les pères contrôlant de ceux qui le sont peu, que l'on note une majorité de pères ou totalement impliqués, c'est-à-dire à la fois stimulants

${ }^{5}$ F/S : Frère/Sœur. 
Tableau 3

Répartition des types de relations père/cadet selon le sexe de la dyade.

\begin{tabular}{|c|c|c|c|c|c|c|c|c|c|c|}
\hline \multicolumn{11}{|c|}{ Composition sexuée de la dyade } \\
\hline Relations & $\mathrm{F}-\mathrm{F}$ & $\%$ & $\mathrm{~F}-\mathrm{G}$ & $\%$ & G-F & $\%$ & $\mathrm{G}-\mathrm{G}$ & $\%$ & Total & $\%$ \\
\hline $\mathrm{S}+\mathrm{C}-$ & 4 & 22 & 2 & 13 & 2 & 17 & 2 & 17 & 10 & 18 \\
\hline$\%$ relation & 40 & & 20 & & 20 & & 20 & & 100 & \\
\hline $\mathrm{S}+\mathrm{C}+$ & 8 & 44 & 6 & 40 & 1 & 8 & 4 & 33 & 19 & 33 \\
\hline$\%$ relation & 42 & & 32 & & 5 & & 21 & & 100 & \\
\hline $\mathrm{S}-\mathrm{C}-$ & 6 & 33 & 4 & 27 & 5 & 42 & 3 & 25 & 18 & 32 \\
\hline$\%$ relation & 33 & & 22 & & 28 & & 17 & & 100 & \\
\hline $\mathrm{S}-\mathrm{C}+$ & 0 & 0 & 3 & 20 & 4 & 33 & 3 & 25 & 10 & 18 \\
\hline$\%$ relation & 0 & & 30 & & 40 & & 30 & & 100 & \\
\hline Total & 18 & 100 & 15 & 100 & 12 & 100 & 12 & 100 & 57 & 100 \\
\hline$\%$ & 32 & & 26 & & 21 & & 21 & & 100 & \\
\hline
\end{tabular}

$\mathrm{S}$ : stimulation; $\mathrm{C}$ : contrôle ; F : fille; G : garcon.

et contrôlants pour leurs cadets $(\mathrm{S}+\mathrm{C}+)(32 \%)$, ou totalement désengagés, c'est-à-dire à la fois non stimulants et non contrôlants $(\mathrm{S}-\mathrm{C}-)(32 \%)$.

La répartition de l'attachement père-enfant selon le sexe des enfants apporte des éléments intéressants (Tableau 3).

En effet, on retrouve $53 \%$ des relations père-cadet « sécurisantes » $(\mathrm{S}+\mathrm{C}+)$ dans les dyades où le cadet est un garçon (32\% dans les dyades mixtes et $21 \%$ dans les dyades unisexes). Il ressort également que $60 \%$ des relations père-cadet dites «contrôlantes » $(\mathrm{S}-\mathrm{C}+)$ se retrouvent dans les dyades où le cadet est un garçon (30\% dans les dyades unisexes et $30 \%$ dans les dyades mixtes). En revanche, $74 \%$ des relations père-cadet « sécurisantes » $(\mathrm{S}+\mathrm{C}+)$ sont observées dans les dyades dont l'aîné est une fille (42\% dans les dyades unisexes et $32 \%$ dans les dyades mixtes). Quant aux relations père-cadet « contrôlantes » $(\mathrm{S}-\mathrm{C}+)$, elles concernent $70 \%$ des dyades où l'aîné est un garçon (30\% des dyades unisexes et $40 \%$ des dyades mixtes). Cependant, une analyse de variance en posthoc ne fait pas ressortir de lien significatif entre le type de relation père-cadet et le sexe de la dyade $\left(\varphi^{6}=4,08: p=0,394\right)$.

\subsubsection{Les relations fraternelles}

Telle que mentionnée dans le Tableau 4, une majorité de relations fraternelles sont ambivalentes, c'est-à-dire empreintes à la fois de coopération et d'opposition (68\%). Si on ajoute à ces relations ambivalentes les relations fraternelles consensuelles (19\%), qui, elles aussi ont un niveau de coopération élevée, ce ne sont pas moins de $87 \%$ des relations fraternelles qui révèlent une coopération importante. Les relations fraternelles empreintes de rivalités et de conflits (cinq dyades fraternelles sur 57) et celles caractérisées par une «faible implication » (deux dyades sur 57) sont minoritaires.

Les relations fraternelles consensuelles et ambivalentes se retrouvent, pour un tiers d'entre elles à chaque fois, dans les familles où le père est, ou bien à la fois stimulant et contrôlant, ou bien ni stimulant ni contrôlant. De même, les relations fraternelles conflictuelles sont, pour plus de la moitié d'entre elles, issues de familles où le père est contrôlant et non stimulant, l'autre moitié se répartissant, de manière égale, entre les pères stimulants et contrôlants, ou ni stimulants, ni contrôlants.

${ }^{6}$ Le coefficient Phi $\varphi$ est obtenu en divisant la valeur du $\mathrm{Khi}^{2}$ par l'effectif total et en prenant la racine carrée. 
Tableau 4

Q5 Nombre de relations fraternelles selon leur type et les types de relations père-cadet.

\begin{tabular}{|c|c|c|c|c|c|c|}
\hline \multirow[b]{2}{*}{ Relations fraternelles } & \multirow[b]{2}{*}{$n$} & \multirow[b]{2}{*}{ Pourcentage } & \multicolumn{4}{|c|}{ Relations père-cadet } \\
\hline & & & $\mathrm{S}+\mathrm{C}+$ & $\mathrm{S}-\mathrm{C}-$ & $\mathrm{S}+\mathrm{C}-$ & $\mathrm{S}-\mathrm{C}+$ \\
\hline Consensuelles $(\mathrm{C}+\mathrm{O}-)$ & 11 & 19 & 4 & 4 & 2 & 1 \\
\hline Ambivalentes $(\mathrm{C}+\mathrm{O}+)$ & 39 & 68 & 13 & 14 & 7 & 5 \\
\hline Conflictuelles $(\mathrm{C}-\mathrm{O}+)$ & 5 & 9 & 1 & 1 & 0 & 3 \\
\hline Faible implication $(\mathrm{C}-\mathrm{O}-)$ & 2 & 4 & 1 & 0 & 1 & 0 \\
\hline Total & 57 & 100 & 19 & 19 & 10 & 9 \\
\hline
\end{tabular}

C : cooperation; O : opposition; $\mathrm{S}$ : stimulation; C : contrôle.

Tableau 5

Q6 Test de Tukey de comparaisons multiples de moyennes de coopération cadet/aîné selon la configuration sexuée des dyades.

\begin{tabular}{lllllcrr}
\hline & Coopération & $n$ & Écart-type & 1 & 2 & 3 & 4 \\
\hline 1. Fille-Fille & 60,56 & 18 & 4,51 & - & $\mathbf{5 , 2 2}^{\circ}$ & 0,72 & 2,81 \\
2. Fille-Garçon & 55,33 & 15 & 7,47 & $\mathbf{5 , 2 2}^{\circ}$ & - & $-4,50$ & $-2,42$ \\
3. Garçon-Fille & 59,83 & 12 & 4,53 & $-0,72$ & 4,50 & - & 2,08 \\
4. Garçon-Garçon & 57,75 & 12 & 7,45 & $-2,81$ & 2,42 & $-2,08$ & -
\end{tabular}

Q7 Les chiffres indiqués correspondent, dans le test de Tukey, aux différences entre les moyennes. Note: * $p<0,05$, ** $p<0,01$, $* * * p<0,001 ;{ }^{\circ} p=0,08$.

\subsubsection{Coopération fraternelle en fonction de l'écart d'âge}

L'Anova montre une légère tendance de l'effet de la variable écart d'âge sur le score de coopération : $\mathrm{F}(1,54)=2,649 ; p=0,08$.

Pour savoir dans quel sens va cet effet, nous avons procédé à un contraste a priori ${ }^{7}$. Nous nous attendons à ce qu'en dessous de deux ans et au-dessus de quatre ans, les scores de coopération soient plus élevés qu'entre deux et quatre ans. Les résultats montrent que les moyennes de scores de coopération obtenus par les enfants, dont l'écart d'âge est inférieur à deux ans $(M=60,03)$, diffèrent significativement de celles obtenues par les enfants dont l'écart d'âge est compris entre deux et quatre ans $(\mathrm{M}=56,68): \mathrm{t}(54)=1,970 ; p<0,05$.

Ce résultat n'est pas retrouvé avec les écarts d'âge supérieurs à quatre ans, en raison du peu de sujets dans ce groupe ( $n=3$ dyades).

\subsubsection{Coopération fraternelle en fonction de la composition sexuée}

Nous avons procédé ici à un test de Tukey de comparaisons multiples des moyennes.

Comme l'indique le Tableau 5, il n'y a pas de différence significative de coopération fraternelle entre les diverses dyades selon leur composition sexuée. Seule une tendance est observée entre les scores de coopération des dyades Fille-Fille $(M=60,56)$ et ceux des dyades Fille-Garçon ( $\mathrm{M}=55,33$ ), $p=0,08$ (Tableau 6).

\subsubsection{Coopération fraternelle en fonction de la taille de la fratrie}

Aucune différence significative de coopération fraternelle n'est trouvée selon la taille de la fratrie.

7 Il s'agit des confirmations empiriques des hypothèses suscitées par la théorie, sur la comparaison des moyennes. 
Tableau 6

Test $t$ de comparaison des moyennes de coopération cadet/aîné selon l'écart d'âge de la dyade aîné-cadet $(n=57)$.

\begin{tabular}{|c|c|c|c|c|c|c|}
\hline Écart d'âge & $n$ & Coopération & Écart-type & 1 & 2 & 3 \\
\hline 1. $<2$ ans & 32 & 60,03 & 5,89 & - & $1,97 *$ & \\
\hline 2. entre 2 et 4 ans & 22 & 56,68 & 6,71 & $1,97 *$ & - & 0,62 \\
\hline 3. $>4$ ans & 3 & 54,33 & 2,31 & 0,62 & - & \\
\hline
\end{tabular}

Note: $* p<0,05, * * p<0,01, * * * p<0,001$.

\subsection{Lien entre activation ou (attachement) père-cadet et qualité des relations fraternelles}

Tableau 7.

\subsubsection{Effet de la stimulation paternelle sur la coopération fraternelle}

L'analyse de la variance univariée indique une différence significative entre le fait que le père soit stimulant, ou non, et le score de coopération fraternelle obtenu. En effet, les cadets de pères stimulants obtiennent un score de coopération avec leur aîné(e) $(M=59,90)$ significativement supérieur à celui des cadets de pères non stimulants $(\mathrm{M}=56,93), \mathrm{F}(1,53)=3,84 ; p<0,05$.

Les contrastes a priori ne montrent cependant aucun effet significatif entre les moyennes de coopération des cadets de pères stimulants/contrôlants $(S+C+)(M=60,05)$ et celles des cadets de pères non stimulants et non contrôlants $(S-C-)(M=58,11)$. De même, aucun effet significatif n'a été trouvé entre les moyennes de coopération des cadets de pères stimulants/contrôlants $(\mathrm{S}+\mathrm{C}+)$ $(M=60,05)$ et celles des cadets de pères stimulants et non contrôlants $(S+C-)(M=59,60)$. Cela signifie que, lorsque les pères sont stimulants, peu importe le niveau de contrôle qu'ils exercent, aucun effet n'est observé sur le niveau de coopération entre le cadet et son aîné(e).

Ayant vu que la stimulation avait, dans certaines conditions, un effet sur le degré de coopération entre les enfants, nous avons essayé d' affiner ces résultats en vérifiant si les stimulations par rapport aux pairs et/ou par rapport aux adultes avaient elles aussi un effet positif sur le degré de coopération des enfants. Les résultats ne montrent aucun effet significatif ni entre la stimulation par rapport aux pairs et le score de coopération $(\mathrm{F}[1,54]=0,973, p=0,328)$, ni entre la stimulation par rapport aux adultes et le score de coopération $(\mathrm{F}[1,54]=1,77, p=0,246)$.

Si nous prenons maintenant en considération la composition sexuée de la dyade fraternelle (Tableau 3), nous observons qu'il y a davantage de relations d'activation $(\mathrm{S}+\mathrm{C}+$ ) dans celles qui

Tableau 7

Test $t$ de comparaison des moyennes de coopération cadet/aîné selon le type de relation père-cadet $(n=57)$.

\begin{tabular}{|c|c|c|c|c|c|c|c|c|c|c|c|}
\hline & \multicolumn{11}{|c|}{ Valeurs du $t$} \\
\hline & $n$ & M & ET & 1 & 2 & 3 & 4 & 5 & 6 & 7 & 8 \\
\hline 1. $\mathrm{S}+$ & 29 & 59,90 & 6,08 & - & $3,84 *$ & 1,20 & & & & & \\
\hline 2. $\mathrm{S}-$ & 28 & 56,93 & 6,30 & $3,84^{*}$ & - & 1,20 & & & & & \\
\hline 3. $\mathrm{C}+$ & 29 & 58,24 & 7,10 & 1,20 & 1,20 & - & 0,69 & & & & \\
\hline 4. $\mathrm{C}-$ & 28 & 58,64 & 5,51 & & & 0,69 & - & & & & \\
\hline 5. $\mathrm{S}+\mathrm{C}+$ & 19 & 60,05 & 6,82 & & & & & - & $-2,17 *$ & 0,95 & $-0,19$ \\
\hline 6. $\mathrm{S}-\mathrm{C}+$ & 10 & 54,80 & 6,61 & & & & & $-2,17 *$ & - & 1,35 & $-1,73^{\circ}$ \\
\hline 7. $\mathrm{S}-\mathrm{C}-$ & & 18 & 58,11 & 5,98 & & & & 0,95 & 1,35 & - & 0,61 \\
\hline 8. $\mathrm{S}+\mathrm{C}-$ & 10 & 59,60 & 4,70 & & & & & $-0,19$ & $-1,73^{\circ}$ & 0,61 & - \\
\hline
\end{tabular}

M : moyenne de coopération, ET : écart-type. Note: $* p<0,05, * * p<0,01, * * * p<0,001,{ }^{\circ} p=0,08$. 
comportent un cadet : en effet, on observe $53 \%$ de relations d'activation lorsque le cadet est un garçon alors qu'elles ne représentent que $47 \%$ lorsqu'il s'agit d'une fille (Tableau 3). Il ne nous a cependant pas été possible d'établir de lien significatif entre les types de relations père-cadet et le sexe des cadets $\left(\mathrm{X}^{2}=1,588, \mathrm{ddl}=3, p=0,662\right.$ au seuil de $\left.5 \%\right)$.

\subsubsection{Effet du contrôle paternel sur la coopération fraternelle}

Les résultats indiquent que chez les pères contrôlants, les moyennes de coopération obtenues par leurs cadets sont significativement plus élevées lorsque les pères sont également stimulants $(\mathrm{M}=60,05)$ que lorsque les pères ne le sont pas $(\mathrm{M}=54,80), \mathrm{F}(1,53)=2,17: p<0,05$.

On ne trouve, par ailleurs, aucune différence significative entre les moyennes de coopération des cadets des pères contrôlants $(M=58,24)$ et des pères non contrôlants $(M=58,64)$.

\section{Discussion}

Tout en restant prudents quant à la généralisation des résultats obtenus à partir d'un échantillon restreint, il apparaît que l'attachement père-enfant ou la relation d'activation, telle que l'évoque Paquette (2004), ce lien affectif qui se tisse entre le père et son enfant dans ce contexte particulier qui concerne la situation de jeux plutôt que celle des soins pour la mère, peut être un des processus pouvant expliquer la qualité des relations entre des frères et sœurs. Bien sûr, ce n'est pas un processus exclusif, car comme analysé dans l'approche systémique, la fratrie constitue un faisceau de relations, elles-mêmes incluses dans la toile des relations familiales... relations, elles-mêmes, tributaires des caractéristiques personnelles de chaque membre de la famille. Ces caractéristiques sont non seulement structurelles, comme la composition sexuée de la fratrie (Abramovitch et al., 1980) ou la taille de celle-ci (Camdessus, 1998), mais aussi individuelle, comme le tempérament de chaque membre (Brody et al., 1996), ou encore psychiques, comme, par exemple, les identifications des parents par rapport à leur propre rang dans leur fratrie (Bourguignon, 1999). Toutes ces variables façonnent chaque individu et sa relation avec «l'autre familial» et rendent complexe l'étude des liens familiaux dans leur ensemble. Notre étude éclaire cependant l'interdépendance de deux variables: le lien père-cadet et la relation cadet-aîné.

Après avoir discuté les relations d'activation père-cadet, d'une part, et les relations cadet-aîné, d'autre part, nous nous pencherons sur les résultats obtenus sur le lien entre ces deux variables.

\subsection{Lien d'attachement ou d'activation père-cadet}

Il est intéressant de noter qu'il existe un peu plus d'activation «sécurisée » $(\mathrm{S}+\mathrm{C}+)-$ bien que ce lien ne soit pas significatif - dans les fratries où le cadet est un garçon que dans les fratries où le cadet est une fille. Cela pourrait s'expliquer par le fait que les jeux physiques, comme celui de «simulacre de bataille», sont plus fréquents entre le père et son fils et que c'est dans ce contexte d'interactions stimulantes que se crée le lien d'attachement père-enfant, précisé ici en termes d'activation.

Le croisement du type de relation existant entre le père et son cadet (stimulation/contrôle) avec le sexe de la dyade ne nous permet pas de considérer que la composition sexuée de la fratrie joue un rôle dans la relation entre le père et le cadet. D'autres études, sur des échantillons plus importants, devront venir confirmer ces résultats préliminaires. Le fait que nous n'ayons pas mesuré l'attachement de l'aîné à son père ne nous permet pas de formuler ici d'hypothèse concernant, par exemple, un effet de compensation possible que le cadet (fille ou garçon) mettrait 
en place dans sa relation fraternelle avec son aînée suite à une faible implication de son père auprès de la dite aînée, hypothèse qu'il nous paraît intéressant de vérifier.

Le plus faible taux d'attachement «sécurisé » père-cadet apparaît dans les dyades où l'aîné est un garçon et la cadette une fille: il serait particulièrement intéressant de mesurer aussi l'attachement de l'aîné à son père. En cas d'attachement sécurisé de l'aîné «garçon» à son père, cela pourrait signifier, par exemple, que le père favoriserait sa relation envers son aîné au dépend de sa cadette. La majorité de relations de «faible implication» $(\mathrm{S}-\mathrm{C}-$ ) retrouvée pour ce type de dyade (aîné garçon-fille cadette) pourrait en être la confirmation.

En ce qui concerne la relation de « contrôle » $(\mathrm{S}-\mathrm{C}+)$ du père envers son cadet, on s'aperçoit que les filles cadettes en bénéficient lorsque leur aîné est un garçon alors que cette relation est inexistante lorsque l'aînée est une fille. Un aîné perçu comme difficile par le père demandant davantage de contrôle, pourrait expliquer que la fille cadette subisse, au moins aux dires de son père, ce même contrôle. Nous devrions alors retrouver ces résultats dans les dyades où le cadet est un garçon, or les résultats indiquent autant de relations « de contrôle pur » père-cadet $(\mathrm{S}-\mathrm{C}+)$ lorsque l'aînée est une fille que lorsque c'est un garçon. Une autre explication serait que les exigences du père envers son fils (que l'on ne retrouverait pas chez la fille cadette) soient à l'origine d'un contrôle plus élevé et se répercutent sur l'ensemble de la fratrie, filles et/ou garçons. Cela est confirmé par le fait que l'on retrouve cette relation de «contrôle », dans des proportions quasiment équivalentes, dans les trois types de dyades comportant un garçon (garçon-garçon, garçon-fille, fille-garçon). Ce type d'exigences et d'attentes du père envers son fils seraient alors intéressantes à approfondir dans des recherches ultérieures.

Les études futures devront considérer simultanément les relations d'attachement de l'aîné et du cadet envers leur père, afin de pouvoir vérifier les hypothèses émises ci-dessus.

De nombreux éléments structurant la constitution de ces relations, il est difficile d'isoler telle ou telle variable pour établir un lien causal, la complexité des relations familiales et de leurs influences mutuelles invite plus à un modèle multifactoriel incluant des variables médiatrices et modératrices que des variables en lien causal unidirectionnel. Rappelons que, dans la perspective systémique, les autres sous-systèmes familiaux non considérés dans notre étude, ici les relations entre le père et la mère, entre la mère et chacun de ses enfants, entre le père et éventuellement les autres enfants de la fratrie, entre l'aîné et le cadet avec les autres frères et sœurs, etc., entrent en jeu et ont chacun une influence sur les autres relations.

\subsection{Les relations fraternelles}

En ce qui concerne les relations fraternelles, on note une majorité de relations fraternelles «ambivalentes », dans lesquelles, sentiments de coopération et d'opposition se côtoient. Nous pensons pouvoir interpréter ce résultat de par l'âge de notre population de cadets, qui se situe entre quatre et six ans, période œdipienne, empreinte de sentiments ambivalents, à la fois à l'égard du parent de sexe opposé, mais aussi de ce rival fraternel (Lacan, 1938). Mais, si l'on considère les relations fraternelles des autres enfants qui ont eux aussi exprimé de forts sentiments de coopération, mais sans opposition, ce sont près de neuf relations cadet-aîné sur dix qui sont imprégnées de ce sentiment de coopération élevée (Tableau 4), ce qui démontre que la coopération reste une dimension importante dans la relation fraternelle d'enfants d'âge scolaire.

Les résultats sur les variables structurelles de la fratrie rejoignent, en partie, ceux retrouvés jusqu'à présent dans la littérature scientifique. Comme Almodovar (1981) et Angel (1996), nous pouvons dire que les enfants ayant un écart d'âge de moins de deux ans s'entendent mieux que les frères et sœurs ayant entre deux et quatre ans d'écart. Notre faible population d'enfants 
ayant un écart d'âge supérieur à quatre ans ne nous permet pas d'émettre de conclusions sur ce sous-groupe. De même, pour la composition sexuée de la fratrie, notre étude confirme les résultats d'Abramovitch, Corter et Pepler (1980) concernant les dyades unisexes de filles plus harmonieuses que les dyades mixtes dont une fille est l'aînée. Ces résultats sont contraires à ceux trouvés par Stewart (1983) pour lequel la mixité tempérerait les rivalités fraternelles ou encore à ceux de Dunn et Kendrick (1979) qui trouvent plus d'interactions négatives dans les dyades unisexes. Outre une explication par les différences d'âges des échantillons (par exemple, enfants plus jeunes dans l'étude de Dunn et al., 1979), ces conclusions confirment la difficulté d'isoler l'une ou l'autre des variables structurelles de la fratrie. Aucun résultat significatif n'a, en revanche, été trouvé pour les dyades de garçons et les dyades mixtes dont l'aîné est un garçon. Les résultats concernant la taille de la fratrie et son effet sur la qualité des relations fraternelles n'ont pas été confirmés. Ainsi, nous ne pouvons conclure, comme Camdessus (1998), que les enfants, issus de familles de trois enfants et plus, profitent de la multiplication des possibilités d'alliance pour augmenter leur degré de coopération entre eux. Il serait intéressant pour les études futures de prendre en compte cette donnée.

\subsection{Lien entre attachement/activation père-cadet et relations fraternelles entre le cadet et l'aîné}

Nos résultats indiquent que seules une relation d'activation avec de la stimulation et du contrôle de la part du père sur son enfant et une relation de faible implication sans stimulation, ni contrôle, ont un effet bénéfique sur la qualité des relations fraternelles. Ces résultats sont contradictoires mais révèlent le lien fort qui unit la qualité de la relation d'un enfant à son père et celle qui l'unit à son frère ou sa sœur. La présence possible à la fois d'un processus spillover ou d'un processus compensatoire entre ces deux dyades souligne l'importance de ne pas les étudier séparément. Par ailleurs, un père uniquement stimulant est également préférable à un père uniquement contrôlant dans la mesure où ce dernier incite à une moindre coopération entre ses enfants.

L'attachement père-cadet a un effet positif sur les relations cadet-aîné puisqu'à contrôle élevé égal, c'est le score de stimulation qui agit sur celui de coopération alors qu'à contrôle bas égal, le score de stimulation n'a aucun effet sur celui de coopération. Ainsi, il ne s'agit pas que le père soit uniquement stimulant envers son cadet pour que celui-ci connaisse de bonnes relations avec son aîné, encore faut-il qu'il soit contrôlant et qu'il suscite donc une exploration sécurisée.

Notons également que la stimulation du père envers son cadet doit être transversale, c'est-àdire concerner tous les domaines, que ce soit sur le plan cognitif, affectif ou social. En effet, et contrairement à nos attentes, l'analyse affinée du lien entre la stimulation du cadet par le père et la moyenne de coopération du cadet envers son aîné, ne met pas en évidence une primauté de la stimulation sociale (envers les pairs ou envers les adultes) sur les autres stimulations (cognitive et affective). Ces résultats contredisent l'affirmation de Volling et Belsky (1992), selon lesquels la stimulation envers les pairs et les adultes, ou stimulation sociale, expliquerait davantage le degré de coopération élevée entre les frères et sœurs.

Les résultats, ne révélant aucune différence significative entre les scores de coopération des cadets « sécurisés » et ceux des cadets dont le père n'est ni stimulant ni contrôlant, permettent de soutenir l'hypothèse émise par Coutu et al. (1995), qu'un processus de compensation se met en place dans la fratrie et que le peu d'implication du père peut être compensée par une plus forte implication des frères et sœurs dans leurs relations fraternelles. Ce résultat intéressant s'oppose 
au processus de spillover, que l'on peut traduire par «effet de contagion», mis en évidence par plusieurs auteurs (Margolin et al., 1996; Erel et Burman, 1995) et qui consiste à dire que les affects et sentiments d'un sous-système familial se «propagent» dans un autre sous-système familial. Ainsi, d'après cette hypothèse, une faible implication du père se propagerait dans la fratrie et rendrait les relations des frères et sœurs plus indifférentes les uns envers les autres. Notre résultat précise le rôle actif des enfants qui peuvent mettre en place, dans leur entourage direct, des conditions propres à leur assurer un environnement affectif équilibré, malgré une «défaillance» des adultes qui en ont la charge (Minuchin, 1974). Ils semblent compenser en effet ici la faible implication du père par une meilleure coopération fraternelle. Il serait intéressant de vérifier dans des études futures le rôle de la période développementale dans ce processus de compensation.

Par ailleurs, les différences significatives trouvées entre le degré de coopération cadet/aîné de père «contrôlant» $(\mathrm{S}-\mathrm{C}+)$ et le degré de coopération cadet/aîné de père «stimulant » $(\mathrm{S}+\mathrm{C}-)$ révèlent que les enfants s'entendent mieux lorsque le cadet est stimulé par son père plutôt qu'uniquement contrôlé par lui. Ces résultats rejoignent ceux de Volling et Belsky (1992) qui avaient montré qu'une interaction prosociale entre l'aîné et le cadet était peu fréquente dans les familles où le père était intrusif et peu impliqué. On peut également rapprocher ces résultats de ceux de Paquette et al. (2000), pour qui les pères autoritaires et peu chaleureux sont moins enclins à jouer physiquement avec leurs enfants. . . et donc a priori à développer les compétences sociales qui résultent de ces interactions.

\section{Conclusion}

Nos résultats ont démontré que les deux axes stimulation et contrôle paternels qui définissent, pour Paquette (2004), la relation d'attachement/activation de l'enfant envers son père, étaient nécessaires à l'enfant, et qu'ils avaient un effet positif sur les relations horizontales qui se vivent au sein du groupe fraternel.

La focalisation sur le cadet, opérée dans cette recherche, plutôt que sur l'aîné, comme le fait l'ensemble des études concernant aussi bien l'attachement que les relations fraternelles, se trouve confortée par les résultats obtenus mettant en exergue le rôle que pourrait jouer le sexe de l'aîné dans la formation de l'attachement du cadet à son père.

Notre étude comporte cependant quelques limites qu'il convient de mentionner. D'une part, des précautions doivent être prises pour ne pas généraliser ces résultats avant que d'autres études les aient répliqués avec une population plus importante. D'autre part, nous avons étudié des pères issus de milieu social favorisé, vivant en couple, qui représentent, d'après l'INSEE (2003), environ un cinquième des pères français, et, il serait intéressant de reproduire cette étude sur des pères issus de différents milieux socioéconomiques.

Enfin, si notre étude s'est centrée sur une relation fraternelle particulière, celle du cadet envers son aîné, elle n'a pas permis de couvrir l'ensemble de cette "polyade » que constitue la famille. L'intérêt d'un travail sur l'ensemble des autres interrelations de la famille, c'est-à-dire sur toutes les relations mutuelles au sein de la fratrie, ainsi que celles incluant à la fois, le père, la mère, et leurs relations avec leurs enfants, le couple coparental et conjugal, s'avère de plus en plus nécessaire pour obtenir une compréhension plus fine de la qualité de l'attachement et de son rôle dans la qualité des relations intrafamiliales. Cette recherche constitue une des étapes nécessaires pour atteindre cet objectif et ainsi permettre une approche systémique de l'attachement répondant également à la question de l'influence de la dynamique familiale sur la qualité de l'attachement parent-enfant. 
Références non citées

\author{
Bowlby (1978), Dubeau et Moss (1998), Minuchin (1998), Troupel et Zaouche-Gaudron \\ (2006).
}

\title{
Références
}

Abramovitch, R., Corter, C., Pepler, D.J., 1980. Observations of mixed-sex dyads. Child Dev 51 (4).

Almodovar, J.P. (1981). Les expériences fraternelles dans le développement de l'enfant. In: M. Soulé (Ed.), Frères et Sœurs. Paris: ESF.

Angel, S., 1996. Des frères et sœurs. Les liens complexes de la fraternité. Robert Laffont, Paris.

Bosso, O.R., 1986. Attachment quality and sibling relations: responses of anxiously/avoidant and securely attached 18to 32-month-old firstborns toward their second-born siblings. Dissertation Abstracts International 47 (3-B), 1293.

Bourguignon, O., 1999. Le Fraternel. Dunod, Paris.

Bowlby, J. (1978). Attachment and loss: Volume I: Attachment, 1969. Traduction française : Kalmanovitch (Jeannine). Attachement et Perte, vol.1, L'attachement. Paris PUF.

Brody, G.H., Stoneman, Z., Gauger, K., 1996. Parent-child Relationships, Family Problem-solving Behavior, and Sibling Relationship Quality: The Moderating Role of Sibling Temperaments. Child Development 67, 1289-1300.

Camdessus, B., 1998. La fratrie méconnue-Liens de sang, liens du cœur. ESF, Paris.

Coutu, S., Provost, M., Pelletier, D. (1995). Relation mère-enfant et qualité des interactions fraternelles chez des enfants d'âge préscolaire. In: http://www.cpa.ca/cjbnex/1996/ful_coutu.html.

Cronbach, L.J., 1951. Coefficient alpha and the internal structure of tests. Psychometrika 16 (3), 297-334.

Dubeau, D., Moss, E., 1998. La théorie de l'attachement résiste-t-elle au charme des pères? Approche comparative des caractéristiques maternelles et paternelles durant la période d'âge préscolaire de l'enfant. Enfance 3, 82-102.

Dunn, J., Kendrick, C., 1979. Interaction between young siblings in the context of family relationships. In: Lewis, M., Rosenblum, C. (Eds.), The Child and his Family. Plemun, New York.

Erel, O., Burman, B., 1995. Interrelatedness of Marital Relations and Parent-Child Relations: A Meta-Analytic Review. Psychol Bull 118 (1), 108-132.

Forman, E.M., Davies, P.T., 2005. Assessing children's appraisals of security in the family system: the development of the Security in the Family System scales (SIFS). Assoc Child Psychol Psychiatry 46 (8), 900-916.

Grossmann, K.E., Grossmann, K., Fremmer-Bombik, E., Kindler, H., Scheuerer-Englisch, H., Zimmermann, P., 2002. The uniqueness of the Child-Father attachment relationship: Father's sensitive and challenging play as a pivotal variable in a 16-year longitudinal study. Soc Dev 3, 307-331.

Hill, J., Fonagy, P., Safier, E., Sargent, J., 2003. The ecology of attachment in the family. Fam Process 42 (2), 205-221.

Kaës, R., 1993. Le complexe fraternel, aspects de sa spécificité. Topiques 51, 5-42.

Kozlowska, K., Lesley, H., 2002. The network perspective: An integration of attachment and family systems theories. Fam Process 41 (3), 285-312.

Kromelow, S., Harding, C., Touris, M., 1990. The role of the father in the development of the stranger sociability during the second year. Am J Orthopsychiatry 60 (4), 521-530.

Lacan, J. (1938). Les complexes familiaux dans la formation de l'individu : essai d'analyse d'une fonction en psychologie. Paris: Navarin. (dernière édition : 1984).

Lamb, M.E., 1977. The development of mother-infant and father-infant attachments in the second year of life. Dev Psychol 13 (6), 637-648.

Lamb, M.E., 1996. The role of the father in child development, 3rd ed. Wiley, New York.

Le Camus, J., Labrell, F., Zaouche-Gaudron, C., 1997. Le rôle du père dans le développement du jeune enfant. Nathan, Noisy-le-Grand.

Le Camus, J., 2000. Le vrai rôle du père. Odile Jacob, Paris.

Margolin, G., Christensen, A., John, R., 1996. The continuance and spillover of everyday tensions in distressed and nondistressed families. J Fam Psychol 10 (3), 304-321.

Marvin, R.S., Stewart, R.B., 1990. A family system framework for the study of attachment. In: Greenberg, M.T., Chiccetti, D., Cummings, E.M. (Eds.), Attachment in the preschool years: Theory, Research and Intervention. University of Chicago Press, Chicago, pp. 51-86.

Miljkovitch de Heredia, R., Pierrehumbert, B., Turganti, G., Halfon, O., 1998. La contribution distincte du père. Enfance $3,103-115$. 
Minuchin, S. (1998). Familles en thérapie. Oxford, England: Harvard U. Press, 1974. Ramonville Saint-Agne : Erès.

Nugent, J.K., 1991. Cultural and psychological influences on the father's role in infant development. J Marriage Fam 53 (2), 475-485.

Paquette, D., 2004. La relation père-enfant et l'ouverture au monde. Enfance 2, 205-225.

Paquette, D., Bolté, C., Turcotte, G., Dubeau, D., Bouchard, C., 2000. A new typology of fathering: Defining and associated variables. Infant Child Dev 9, 213-230.

4 Paquette, D., Eugène, M.M., Dubeau, D., Gagnon, M.N. (sous presse). Les pères ont-ils des influences spécifiques sur le développement des enfants? In: D. Dubeau, A. Devault, G. Forget (Eds.), Prospère : la paternité du $21^{\mathrm{e}}$ siècle, PUL.

Stewart, R.B., 1983. Sibling attachment relationships: child-infant interactions in the strange situation. Dev Psychol 19 (2), 192-199.

Teti, D.M., Ablard, K.E., 1989. Security of attachment and infant-sibling relationships: a laboratory study. Child Dev 60, 1519-1528.

Troupel, O. (2006). Attachement fraternel, styles de relations et des interactions de tutelle au sein des fratries de jeunes enfants : effet modulateur de la représentation des relations fraternelles de l'aîné. Thèse de Doctorat Nouveau régime. Université de Toulouse-Le Mirail, 2006.

Troupel, O., \& Zaouche-Gaudron, C. (2006). Communication au XXIIIe Colloque du GROFRED «L'enfant et sa famille : interactions et transmissions ». Nancy (France), 26-27 mai.

Volling, B.L., Belsky, J., 1992. The contribution of mother-child and father-child relationships to the quality of sibling interaction: a longitudinal study. Child Dev 63, 1209-1222.

Welz, S. (1984). Analyse der Geschwisterbeziehungen in Familien der Bielefelder Längsschnittuntersuchung (Analysis of sibling relationships in Families of the Bielefeld longitudinal study). Diplomthesis. Universität Regensburg.

Widmer, E., 1999. Les relations fraternelles à l'adolescence. PUF, Paris.

Yogman, M., Kindlon, D., Earls, F., 1995. Father involvement and cognitive/behavioral outcomes of preterm infants. J Am Acad Child Adolesc Psychiatry 34 (1), 58-66.

Zaouche-Gaudron, C., 2002. Le développement social de l'enfant (du bébé à l'enfant d'âge scolaire). Dunod, Paris.

Liens Internet : http://www.INSEE.fr. 